\title{
Cytoreductive surgery and HIPEC in colorectal cancer-did we get hold of the wrong end of the stick?
}

\author{
Can Yurttas · Oliver M. Fisher · Delia Cortés-Guiral · Sebastian P. Haen · Ingmar Königsrainer · \\ Alfred Königsrainer · Stefan Beckert · Winston Liauw · Markus W. Löffler (DD
}

Received: 19 July 2020 / Accepted: 14 September 2020 / Published online: 20 October 2020

(C) The Author(s) 2020

\begin{abstract}
Summary Cytoreductive surgery (CRS) and hyperthermic intraperitoneal chemotherapy (HIPEC) are a multimodal treatment approach combining surgical interventions of varying extent with administration of heated cytostatic drugs flushed through the abdominal cavity. Hitherto, this treatment has been popular for peritoneal metastasis (PM), e.g. from colorectal cancer (CRC). Recent randomized controlled trials (RCT) question the benefit of HIPEC in its present form for CRC treatment and raise fundamental issues,
\end{abstract}

C. Yurttas · I. Königsrainer · A. Königsrainer · S. Beckert . M. W. Löffler $(\bowtie)$

Department of General, Visceral and Transplant

Surgery, University Hospital Tübingen,

Hoppe-Seyler-Str. 3, 72076 Tübingen, Germany

markus.loeffler@uni-tuebingen.de

\section{O. M. Fisher}

Upper GI and Hepatobiliary Surgery Unit, Royal Prince Alfred Hospital, 50 Missenden Road, 2050 Camperdown, New South Wales, Australia Notre Dame University, School of Medicine, Sydney, 160 Oxford St, 2010 Darlinghurst, New South Wales, Australia

O. M. Fisher · W. Liauw

St George and Sutherland Clinical School, University of New South Wales, Short St, 2217 Kogarah, New South Wales, Australia

\section{Cortés-Guiral}

Department of Surgical Oncology, King Khalid University

Hospital, 66262 Najran, Alkhalidiyyah, Saudi Arabia

\section{S. P. Haen}

Department of Oncology, Haematology and Bone Marrow Transplantation with Section of Pneumology,

University Medical Center Hamburg-Eppendorf,

Martinistr. 52, 20246 Hamburg, Germany

\section{Königsrainer}

Academic Teaching Hospital Feldkirch,

Carinagasse 47, 6800 Feldkirch, Austria eliciting discussions and expert statements regarding HIPEC relevance and interpretation of these results. Unfortunately, such discussions have to remain uninformed, due to the lacking publication of crucial peer reviewed RCT results. Novel basic research aware of HIPEC futility suggests there may be systematic limitations. Innovative modelling approaches for HIPEC may shed light on the reasons for therapeutic failure of frequently used drugs and may lead the way to select

\section{A. Königsrainer · M. W. Löffler \\ German Cancer Consortium (DKTK) and German Cancer Research Center (DKFZ) Partner Site Tübingen, Tübingen, Germany}

A. Königsrainer • M. W. Löffler

Cluster of Excellence iFIT (EXC 2180) "Image-Guided and Functionally Instructed Tumor Therapies", University of Tübingen, Tübingen, Germany

\section{S. Beckert}

Department of General and Visceral Surgery, Schwarzwald-Baar Hospital,

Klinikstr. 11, 78052 Villingen-Schwenningen, Germany

\section{W. Liauw}

Cancer Care Centre, St George Hospital, Gray St, 2217 Kogarah, New South Wales, Australia

\section{W. Löffler}

Interfaculty Institute for Cell Biology, Department of Immunology, University of Tübingen, Auf der Morgenstelle 15, 72076 Tübingen, Germany

Department of Clinical Pharmacology, University Hospital Tübingen, Auf der Morgenstelle 8, 72076 Tübingen, Germany 
better alternatives and/or more rational approaches for the design of HIPEC procedures (e.g. regarding exposure time or temperature). Available evidence strongly supports the notion that CRS is the mainstay for the treatment effects observed in PM from CRC. Unfortunately, HIPEC has become a surrogate for surgical expertise in the field and optimal surgery may therefore outweigh the potentially harmful effects of HIPEC treatment, particularly in lieu of modern systemic chemotherapies. The current situation which frequently is assumed to be deadlocked should be regarded as a challenge to investigate HIPEC with welldesigned prospective clinical trials, potentially even constituting an opportunity for introducing innovative trial designs that solve the multifaceted issues of a very heterogeneous treatment approach.

Keywords PRODIGE 7 - Randomized controlled trial • Oxaliplatin · Peritoneal metastasis · Hyperthermic intraperitoneal chemotherapy

\section{Background}

Metastases from CRC in the peritoneum are associated with a poor prognosis. In about $5 \%$ of CRC patients metastasis remains confined to the peritoneum as the sole site of dissemination [1]. Nonetheless, this disease presentation has been considered incurable and merely amenable to palliative treatment options, including systemic chemotherapy or simply best supportive care [2], due to a presumed peritoneal-plasma barrier preventing cytostatic drug influx into the peritoneum [3] and reservations regarding feasibility and appropriateness of surgery.

Inter alia, in CRC the notion of futility of therapeutic approaches in PM has been challenged with the availability of specialized surgical treatment (i.e. CRS) combined with HIPEC, offering a multimodal treatment approach associated with improved survival prospects [4]. Up to now, no published RCT has established the independent benefit of HIPEC in CRC when added to CRS, and consequently this treatment has usually been offered as a "complete package".

This article aims to summarize the current state and best evidence-base for HIPEC in CRC and discusses its current limitations with an emphasis on recent RCTs and preclinical research with a view to improving future strategies.

\section{Cytoreductive surgery}

It is established that even extensive surgery is safe, feasible and clinically effective for managing PM. Surgical proficiency is a prerequisite, implying relevant training and expertise in complex surgical procedures [5]. In addition, competent patient selection considering the extent and location of metastases is crucial [6], since complete surgical cytoreduction has been shown to translate into improved survival in both CRC and ovarian cancer [4]. Of note, the concept of CRS differs largely from most conventional surgical oncology procedures amongst other things with regard to the radicality of surgery.

Although the treatment may cause considerable morbidity [7], current data suggest the associated risk is comparable with other high-risk procedures in surgical oncology [8] and many centers report procedure-related mortality rates of $\sim 1 \%$ [9]. CRS and HIPEC have therefore become a frequent treatment approach for $\mathrm{PM}$ in various malignancies including CRC and ovarian cancer, challenging a purely palliative treatment pathway.

\section{Hyperthermic intraperitoneal chemotherapy}

CRS is inevitably linked to HIPEC and rarely ever performed without this adjunct, as both treatments have been interconnected from the beginning.

While HIPEC has an intuitive and plausible theoretical rationale, the diversity of protocols used worldwide is overwhelming $[10,11]$. As convincing preclinical evidence to inform clinical practice remains the exception such research frequently post-dates the clinical use of drugs for HIPEC (cf. studies with oxaliplatin [12, 13] or mitomycin C (MMC) [14]).

Therefore, despite the fact that HIPEC has been applied to countless patients after CRS, many fundamental questions remain unresolved. The most commonly administered drugs during HIPEC in CRC have been MMC and oxaliplatin, but also many other drugs and drug combinations are described [11]. Additional factors complicate HIPEC as a therapy, including a wide range of drug concentrations, drug diluents as well as exposure times and temperatures [10]. Due to these inconsistencies and vaguely described HIPEC procedures, uniformity is lacking, and fundamental disagreement remains regarding the standardization of HIPEC protocols [15, 16].

\section{Best evidence from randomized controlled trials}

Central for the establishment of CRS and HIPEC (with MMC for $90 \mathrm{~min}$ ) as a valid treatment option for PM in CRC was a first-ever and thus far the only completed (and published) prospective RCT performed by Verwaal et al. between 1998 and 2001 [17]. Trial results showed prolonged 2-year overall survival (OS) with CRS and HIPEC vs. palliative intravenous (i.v.) chemotherapy alone. Median OS nearly doubled from 12.6 to 22.3 months for the intervention group. However, back then this benefit did not prove sustainable after 5 years. Despite the impressive results, relevant limitations for this trial should be kept in mind:

- An i.v. chemotherapy protocol outdated at time of publication was used for the control group,

- The trial design did not allow to assess the contributions of CRS and HIPEC separately [18], 
- Heterogeneous malignancies were included with different biological behaviors and prognosis.

Nevertheless, this RCT was the cornerstone for introducing this treatment in PM of CRC worldwide and helped to improve patient selection, surgical approaches and ultimately the outcomes for these patients [4].

\section{PRODIGE 7: discussing an unpublished clinical trial}

The PRODIGE 7 trial was subsequently set up years later to assess the survival benefits of CRS and HIPEC with oxaliplatin vs. CRS alone. Unfortunately, this trial has remained unpublished despite representing the most important study in this context. Results have thus far been presented at conferences, initially the Annual Meeting of the American Society of Clinical Oncology (ASCO) in June 2018, while the peer reviewed publication is still awaited [19]. Therefore, all available information deriving from this study needs to be considered prefinal. According to available reports, median OS is about 41 months in both groups (41.7 vs. 41.2 months, hazard ratio $[\mathrm{HR}]=1.00$, 95\% confidence interval [CI] 0.73-1.37, $p=0.995)$ and surgery seems to be of primary relevance for the observed survival benefits. Short-term oxaliplatin HIPEC over $30 \mathrm{~min}$ failed to show OS benefits, but associated complications were reported to be significantly increased. However, neither of the two treatment modalities increased 30-day mortality rates [20].

A central point of criticism that questions basic prerequisites of the PRODIGE 7 trial is the planning and presumptions made for the study beforehand. Accordingly, the sample size estimation was based on the assumption of a median OS gain from 30 to 48 months by the used HIPEC treatment [19]. In retrospect this assumption seems to be overly optimistic, due to the minimal OS differences observed between the two groups, suggesting that the effects of HIPEC have been grossly overestimated, whereas the relevance of radical surgery has been underrated.

In contrast, there is no shortage of less robust data, including case series with complete surgical cytoreduction and HIPEC, where even more remarkable results such as a 5 -year OS rate of $51 \%$ and cure rates of about $16 \%$ have been reported [21], suggesting there may be highly effective protocols available in this difficult disease spectrum.

But, the negative PRODIGE 7 RCT results have fundamentally challenged long-standing CRS and HIPEC practice and fueled discussions and interpretations albeit in the absence of a formal publication [22, 23]. Furthermore, there has been no shortage of consensus statements and expert opinions (e.g. [24]), suggesting how to deal with the findings. The status quo is that PRODIGE 7 cannot be ignored in PM for CRC but a peer reviewed publication is required and eagerly awaited for any valid interpretation.

\section{Is oxaliplatin the wrong drug for HIPEC?}

Meanwhile, HIPEC has entered an identity crisis in the surgical oncology community not only based on the preliminary results of the PRODGE 7 trial [19] but also because further evidence has amounted showing futility in RCTs performed in the adjuvant setting [25].

Aiming to compile evidence to understand the underlying mechanisms for failure of certain HIPEC protocols, our group has recently gathered preliminary evidence (available as a preprint) suggesting that during short-term HIPEC, oxaliplatin is unable to penetrate deep enough into cell layers $(\sim 100 \mu \mathrm{m})$ to cause relevant cell death even in micrometastases [12]. Likewise, Ubink et al. have evaluated clinically used HIPEC protocols in an in vitro CRC organoid model [13], where both MMC and oxaliplatin were shown ineffective to induce robust cell death at commonly attained drug dosages reached during HIPEC in vivo, suggesting there may be more generic issues. Notably all of the assessed CRC organoids showed resistance to HIPEC treatment with oxaliplatin.

Admittedly, this research is simplistic and insufficient to invalidate any of the clinically used HIPEC protocols. However, in conjunction with the RCT results such findings do raise fundamental questions as to the current conduct and role of HIPEC. Equally, the discussion surrounding the pharmacokinetics and -dynamics of any cytotoxic agent for intraperitoneal administration requires enforcement, particularly through robust scientific evidence, given that our knowledge in this field is fairly poor.

\section{How do we go on?}

The vulnerability of HIPEC is based on its broad implementation without prior verification of drug effects and effectiveness applicable to most treatment protocols (including variables such as drug concentration, exposure time, carrier solution, temperature, intra-abdominal pressure, etc.).

Considering both published and unpublished data, many open questions regarding the design and effectiveness of clinically used HIPEC protocols arise. A weakness of CRS and HIPEC was the introduction of a complex compound treatment (cytoreductive surgery, hyperthermia, chemotherapy, abdominal lavage etc.) that does not allow to discern precisely, which of the benefits but also which adverse effects can be ascribed to which component. Here, the PRODIGE 7 trial would have been a crucial step towards scientific integrity and the broad implementation of HIPEC, as well as changes in protocols since the presentation of PRODIGE 7 outside of clinical trial settings has proven a relevant liability. In order to avoid repeating the mistakes of the past it would 
be advisable to reinitiate well-designed preclinical studies and to gradually bridge results from bench to the bedside. Equally, analyzing the process by which HIPEC has been assessed within ovarian cancer and adopting some of the lessons learned from our gynaecological colleagues may equally be advisable [26]

Available evidence not only supports the notion that short-term oxaliplatin HIPEC is ineffective and potentially even harmful for patients but also that HIPEC should be established in a controlled clinical trial environment. New results question the practice of many HIPEC protocols and even long held beliefs e.g. that excessive doses of cytostatic drugs in single application have clinical benefits and they should therefore be rigidly scrutinized. As such, once preclinical studies have identified a robust case for the design of a HIPEC protocol, RCT testing is ideal but logistically difficult. PRODIGE 7 took many years to complete recruitment and after dissemination of the results, achieving international consensus as to appropriate trial designs has proven challenging. One way forward to circumvent the challenges of conducting bespoke RCT would be to establish an international adaptive platform clinical trial protocol in PM from CRC. Such a protocol could establish a control arm comprising CRS for PM in CRC. Multiple parallel or sequential intervention arms could be conducted, including CRS and HIPEC, CRS and early postoperative intra-peritoneal chemotherapy, CRS and normothermic intraperitoneal chemotherapy, CRS and peri-operative chemotherapy, etc. As one intervention has turned out to be superior according to predefined stopping rules, a new standard of care control arm would emerge [27]. Such an approach would potentially be more efficient but could also lead to global harmonization of approaches to HIPEC protocols and benchmarking for the quality and safety of cytoreductive surgery.

\section{Conclusion}

In summary CRC, even in the metastatic setting has become a disease amenable to surgical treatments and the prognosis of patients with PM has considerably improved. In different indications there are multimodal approaches, combining metastasectomy (liver/lung) with systemic chemotherapy that lead to unexpected long-term survival [28, 29]. Against this background it is noteworthy that the PRODIGE 7 trial has also established an impressive median OS of $>41$ months with CRS alone [19]. But, to what extent these results were driven by CRS and/or systemic therapies before and after surgery remains to be elucidated.

For this reason, it should be emphasized that leading experts and scientific societies concerned with peritoneal surface malignancies are currently struggling to provide clear guidelines and HIPEC remains the subject of heavy debate [30]. We therefore believe the standard of care remains CRS, whereas HIPEC should be addressed by a new wave of interest in basic research relating to the intraperitoneal application of chemotherapeutic agents as well as through new, innovative clinical trial designs that will enable us to generate sufficient evidence to identify the most beneficial treatment approach for patients with CRC and peritoneal metastases.

\section{Take home message}

- Long-standing practise of hyperthermic intraperitoneal chemotherapy (HIPEC) in peritoneal metastasis (PM) from colorectal cancer (CRC) has been challenged by new results.

- The overdue publication of crucial randomized controlled trial results in PM from CRC (PRODIGE 7) is eagerly awaited and has contributed to the prevailing uncertainty.

- Complete cytoreductive surgery is the mainstay of treatment with relevant effects, and if feasible, should (still) be considered the standard of care in PM from CRC.

- HIPEC, constituting a very heterogeneous treatment and frequently lacking supportive (preclinical) evidence, remains experimental in $\mathrm{CRC}$ and requires clinical trial evaluation.

- Basic research (e.g. new modelling approaches) and innovative clinical trial designs should be strengthened to help with identification of the most effective treatment approach.

Funding Open Access funding enabled and organized by Projekt DEAL.

Conflict of interest M. W. Löffler and A. Königsrainer have received grant support by RanD S.r.l., a manufacturer of devices and consumables for HIPEC, unrelated to the present work. M. W. Löffler is a co-inventor of several patents owned by Immatics Biotechnologies, and has acted as a consultant and/or advisory board member for Boehringer Ingelheim outside the submitted work. O. M. Fisher reports personal fees from GORE and Fisher \& Paykel Healthcare outside the submitted work. C. Yurttas, D. Cortés-Guiral, S. P. Haen, I. Königsrainer, S. Beckert and W. Liauw declare that they have no competing interests.

Open Access This article is licensed under a Creative Commons Attribution 4.0 International License, which permits use, sharing, adaptation, distribution and reproduction in any medium or format, as long as you give appropriate credit to the original author(s) and the source, provide a link to the Creative Commons licence, and indicate if changes were made. The images or other third party material in this article are included in the article's Creative Commons licence, unless indicated otherwise in a credit line to the material. If material is not included in the article's Creative Commons licence and your intended use is not permitted by statutory regulation or exceeds the permitted use, you will need to obtain permission directly from the copyright holder. To view a copy of this licence, visit http://creativecommons.org/licenses/by/4.0/. 


\section{References}

1. Segelman J, Granath F, Holm T, Machado M, Mahteme H, Martling A. Incidence, prevalence and risk factors for peritoneal carcinomatosis from colorectal cancer. $\mathrm{Br} J$ Surg. 2012;99(5):699-705.

2. Lambert LA. Looking up: recent advances in understanding and treating peritoneal carcinomatosis. CA Cancer J Clin. 2015;65(4):284-98.

3. Jacquet P, Sugarbaker PH. Peritoneal-plasma barrier. CancerTreat Res. 1996;82:53-63.

4. Verwaal VJ, Bruin S, Boot H, van Slooten G, van Tinteren H. 8-yearfollow-up of randomized trial: cytoreduction and hyperthermic intraperitoneal chemotherapy versus systemic chemotherapy in patients with peritoneal carcinomatosis of colorectal cancer. Ann Surg Oncol. 2008;15(9):2426-32.

5. Kusamura S, Gonzalez-Moreno S, Nizri E, Baratti D, Guadagni S, Guaglio M, et al. Learning curve, training program, and monitorization of surgical performance of peritoneal surface malignancies centers. Surg OncolClin N Am. 2018;27(3):507-17.

6. Simkens GA, Rovers KP, Nienhuijs SW, de Hingh IH. Patient selection for cytoreductive surgery and HIPEC for the treatment of peritoneal metastases from colorectal cancer. Cancer Manag Res. 2017;9:259-66.

7. Glockzin G, Ghali N, Lang SA, Schlitt HJ, Piso P. Results of cytoreductive surgery and hyperthermic intraperitoneal chemotherapy for peritoneal carcinomatosis from colorectal cancer. J Surg Oncol. 2009;100(4):306-10.

8. Foster JM, Sleightholm R, Patel A, Shostrom V, Hall B, Neilsen B, et al. Morbidity and mortality rates following cytoreductive surgery combined with hyperthermic intraperitoneal chemotherapy compared with other highrisk surgical oncology procedures. JAMA Netw Open. 2019;2(1):e186847.

9. Kyang LS, Alzahrani NA, Valle SJ, Rahman MK, Arrowaili A, Liauw W, et al. Long-term survival outcomes of cytoreductive surgery and perioperative intraperitoneal chemotherapy: single-institutional experience with 1225 cases. J Surg Oncol. 2019;120(4):794-802.

10. Helderman R, Loke DR, Kok HP, Oei AL, Tanis PJ, Franken N, et al. Variation in clinical application of hyperthermic intraperitoneal chemotherapy: a review. Cancers (Basel). 2019;11(1):78.

11. Yurttas C, Hoffmann G, Tolios A, Haen SP, Schwab M, Königsrainer I, et al. Systematic review of variations in hyperthermic intraperitoneal chemotherapy (HIPEC) for peritoneal metastasis from colorectal cancer. J Clin Med. 2018;7(12):567. https://doi.org/10.3390/jcm7120567.

12. Löffler MW, Seyfried N, Burkard M, Oswald B, Tolios A, Yurttas C, et al. Short-term oxaliplatin exposure according to establishedhyperthermicintraperitoneal chemotherapy (HIPEC) protocols lacks effectiveness in vitro and ex vivo. bioRxiv. 2019; https://doi.org/10.1101/709055.

13. Ubink I, Bolhaqueiro ACF, Elias SG, Raats DAE, Constantinides A, Peters NA, et al. Organoids from colorectal peritoneal metastases as a platform for improving hyperthermic intraperitoneal chemotherapy. Br J Surg. 2019;106(10):1404-14.

14. Klaver YL, Hendriks T, Lomme RM, Rutten HJ, Bleichrodt RP, de Hingh IH. Hyperthermia and intraperitoneal chemotherapy for the treatment of peritoneal carcinomatosis: an experimental study. Ann Surg. 2011;254(1):125-30.

15. Kuijpers AM, Aalbers AG, Nienhuijs SW, de Hingh IH, Wiezer MJ, van Ramshorst B, et al. Implementation of a standardized HIPEC protocol improves outcome for peritoneal malignancy. World J Surg. 2015;39(2):453-60.
16. Turaga K, Levine E, Barone R, Sticca R, Petrelli N, Lambert L, et al. Consensus guidelines from the American society of peritoneal surface malignancies on standardizing the delivery of hyperthermic intraperitoneal chemotherapy (HIPEC) in colorectal cancer patients in the United States. Ann Surg Oncol. 2014;21(5):1501-5.

17. Verwaal VJ, van Ruth S, de Bree E, van Sloothen GW, van Tinteren $\mathrm{H}$, Boot H, etal. Randomized trial of cytoreduction and hyperthermic intraperitoneal chemotherapy versus systemic chemotherapy and palliative surgery in patients with peritoneal carcinomatosis of colorectal cancer. J Clin Oncol. 2003;21(20):3737-43.

18. Markman M. Intraperitoneal hyperthermic chemotherapy as treatment of peritoneal carcinomatosis of colorectal cancer. JClin Oncol. 2004;22(8):1527. author reply 9.

19. Quenet F, Elias D, Roca L, Goere D, Ghouti L, Pocard M, et al. A UNICANCER phase III trial of hyperthermic intraperitoneal chemotherapy (HIPEC) for colorectal peritoneal carcinomatosis (PC): PRODIGE 7. J Clin Oncol. 2018; https://doi.org/10.1200/JCO.2018.36.18 SUPPL.LBA3503.

20. Mohamed F, Kallioinen M, Braun M, Fenwick S, Shackcloth M, Davies RJ, et al. Management of colorectal cancer metastases to the liver, lung or peritoneum suitable for curative intent: summary of NICE guidance. Br J Surg. 2020;107(8):943-5.

21. Elias D, Lefevre JH, Chevalier J, Brouquet A, Marchal F, Classe JM, et al. Complete cytoreductive surgery plus intraperitoneal chemohyperthermia with oxaliplatin for peritoneal carcinomatosis of colorectal origin. JClin Oncol. 2009;27(5):681-5.

22. Ceelen W. HIPEC with oxaliplatin for colorectal peritoneal metastasis: the end of the road? Eur J Surg Oncol. 2019;45(3):400-2.

23. Königsrainer A, Rau B. Cytoreductive surgery (CRS) and hyperthermic intraperitoneal chemotherapy (HIPEC): don't throw the baby out with the bathwater. Pleura Peritoneum. 2018;3(4):20180131.

24. Abboud K, Andre T, Brunel M, Ducreux M, Eveno C, Glehen $\mathrm{O}$, et al. Management of colorectal peritoneal metastases: expert opinion. J Visc Surg. 2019;156(5):377-9.

25. Klaver CEL, Wisselink DD, Punt CJA, Snaebjornsson P, Crezee J, Aalbers AGJ, et al. Adjuvant hyperthermic intraperitoneal chemotherapy in patients with locally advanced colon cancer (COLOPEC): a multicentre, openlabel, randomised trial. Lancet Gastroenterol Hepatol. 2019;4(10):761-70.

26. Vergote I, Harter P, Chiva L. Is there a role for intraperitoneal chemotherapy, including HIPEC, in the management of ovarian cancer? J Clin Oncol. 2019;37(27):2420-3.

27. The Adaptive Platform Trials Coalition. Adaptive platform trials: definition, design, conduct and reporting considerations. Nat Rev Drug Discov. 2019;18(10):797-807.

28. Milosevic M, Edwards J, Tsang D, Dunning J, Shackcloth M, Batchelor T, et al. Pulmonary metastasectomy in colorectal cancer: updated analysis of 93 randomized patients-control survival is much better than previously assumed. Colorectal Dis. 2020; https://doi.org/10.1111/ codi.15113.

29. VäyrynenV,WirtaEV, SeppalaT, Sihvo E, MecklinJP, VasalaK, etal. Incidence and management of patients with colorectal cancer and synchronous and metachronous colorectal metastases: a population-based study. BJS Open. 2020; https://doi.org/10.1002/bjs5.50299.

30. Steffen T, Eden J, Bijelic L, Glatzer M, Glehen O, Goéré D, et al. Patient selection for hyperthermic intraperitoneal chemotherapy (HIPEC) in colorectal cancer patients: consensus on decision making amongst international experts. 
Clin Colorectal Cancer. 2020; https://doi.org/10.1016/j. clcc.2020.06.010.

Publisher's Note Springer Nature remains neutral with regard to jurisdictional claims in published maps and institutional affiliations.
- For latest news from international oncology congresses see: http://www.springermedizin.at/ memo-inoncology 